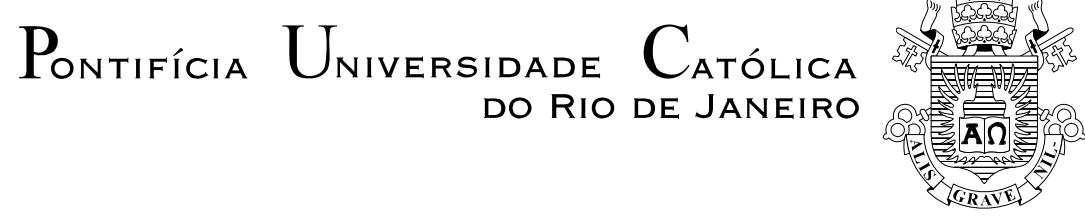

Ronaldo Pedro da Silva

Simulação e Análise do Desempenho de um Laboratório Analítico em um Cenário Auto-sustentável

Dissertação de Mestrado

Dissertação apresentada como requisito parcial para obtenção do grau de Mestre pelo Programa de Pósgraduação em Engenharia Industrial do Departamento de Engenharia Industrial da PUC-Rio.

Orientador: Prof. Eugenio Kahn Epprecht

Rio de Janeiro

Abril de 2007 
Ronaldo Pedro da Silva

\title{
Simulação e Análise do Desempenho de um Laboratório Analítico em um Cenário Auto-sustentável
}

\begin{abstract}
Dissertação apresentada como requisito parcial para obtenção do título de Mestre pelo Programa de PósGraduação em Engenharia Industrial da PUC-Rio. Aprovada pela Comissão Examinadora abaixo assinada.
\end{abstract}

Prof. Eugenio Kahn Epprecht Orientador Departamento de Engenharia Industrial - PUC-Rio

Prof. Leonardo Junqueira Lustosa Departamento de Engenharia Industrial - PUC-Rio

Prof. Roberto Ribeiro de Avillez

Departamento de Ciências dos Materiais e Metalurgia - PUC-Rio

Prof. José Eugenio Leal Coordenador Setorial do Centro Técnico Científico - PUC-Rio 
Todos os direitos reservados. É proibida a reprodução total ou parcial do trabalho sem autorização da universidade, do autor e do orientador.

\section{Ronaldo Pedro da Silva}

Graduou-se em Administração de Empresas no IAG/PUC-Rio em 2004. Trabalhou cerca de 23 anos em ambiente de pesquisa (Centro de Pesquisas da CSN e DCMM/PUC-Rio). Concebeu, em 1997 no Instituto Gênesis, uma empresa spin-off de treinamento em equipamentos e processos com simulação multimídia. Publicou trabalhos em congressos e revistas de caracterização de materiais, engenharia de produção e administração (Brazilian Society for Electron Microscopy, 2001; VIII Latin American Seminar on XRay Analysis Techniques-SARX, 2002; Acta Microscopia, 2002; dois artigos no XXV Encontro Nacional de Engenharia de Produção, 2005 e Revista de Economia e Administração IBMEC, 2006). Atualmente é Supervisor do LabDRX/DCMM/PUC-Rio.

Ficha Catalográfica

Pedro-Silva, Ronaldo

Simulação e análise do desempenho de um laboratório analítico em um cenário auto-sustentável / Ronaldo Pedro-Silva ; orientador: Eugenio Kahn Epprecht. - 2007.

129 f. : il. ; $30 \mathrm{~cm}$

Dissertação (Mestrado em Engenharia Industrial)Pontifícia Universidade Católica do Rio de Janeiro, Rio de Janeiro, 2007.

Inclui bibliografia

1. Engenharia Industrial - Teses. 2. Simulação. 3. Laboratório analítico. 4. Difração de raios-X. 5. Gestão de laboratórios de P\&D. 6. Auto-sustentável. I. Epprecht, Eugenio Kahn. II. Pontifícia Universidade Católica do Rio de Janeiro. Departamento de Engenharia Industrial. III. Título. 
Dedico este trabalho a minha querida esposa, Elvira, como ínfima parcela do reconhecimento por tudo que fez e faz por nossos filhos, Júlia e Pedro, e pela minha vida pessoal e profissional. Obrigado por me fazer e deixar sonhar... 


\section{Agradecimentos}

A Deus, pela existência, oportunidades e saúde;

Ao meu orientador Professor Eugenio Kahn Epprecht, pela objetividade, franqueza, parceria e, principalmente, pela revisão detalhada de todo conteúdo desta dissertação, bem como a confiança depositada na estruturação desta pesquisa;

Ao Professor Roberto Ribeiro de Avillez, pela total liberdade no uso de dados e informações do LabDRX e, particularmente, pela flexibilidade, oportunidades de estudos e aprendizado ao longo destes 10 anos de trabalho na PUC-Rio. Parece se repetir à história entre dois homens, Manuel Bandeira e um jovem chamado Avillez;

A Companhia Siderúrgica Nacional, pelo acesso e utilização do Arena versão 10.0 profissional e, em particular, ao meu tutor neste software, o Eng. Bruno Calazans, pela paciência e disponibilidade;

A minha amiga Professora Terezinha Ferreira de Oliveira, pelas palavras de incentivo e pelo fundamental apoio na fase preliminar do mestrado;

A Professora Maria Angela de Campelo Melo e Professor Nélio Domingues Pizzolato, pela receptividade e orientação na matrícula;

Ao Professor Augusto Sampaio e a Coordenação Central de Pós-Graduação e Pesquisa da PUC-Rio, pela bolsa de isenção VRAC I;

Ao Dr. Valter Rocha dos Santos, pelos conselhos e generosa transmissão de experiências durante o cafezinho diário, além das cobranças "A dissertação está pronta?";

Ao Professores Paulo Dalcol e Fernando Rizzo, pelas orientações acadêmicas e aos colegas funcionário do Departamento de Engenharia Industrial da PUC-Rio Cláudia, Izabel, Fernanda, Eduardo, Celi e Gilvan, além de todos colegas de trabalho do Departamento de Ciência dos Materiais e Metalurgia da PUC-Rio;

Aos amigos do Mestrado: Daniel, Leo, Thiago, Wellington, Jaci, Rosana, Eduardo, Arnaldo, José, Silvio, e, em especial, a Yuri, Tiago, Bruno Estatístico, Bruno, Cristina, Danilo, Ulisses e Aninha "E o churrasco, galera?", além de muitos outros amigos das turmas de 2005 e 2006;

A minha querida esposa, Elvira, pela enorme apoio, incentivo e paciência. Pode marcar a viagem, pois desta vez eu vou; 
Aos melhores filhos do mundo, Pedro e Júlia, pelos momentos de carinho, alegria e diversão.

A amiga Márcia Alonso, pelos inúmeros auxílios e apoio aos estudos de Júlia que, indiretamente, contribuíram com este;

A minha querida e vitoriosa mãe, Tereza Vicença Viana, meus queridos irmãos Marzim e Sandra e a "grande família": Mário, José Horta, Abílio, Patrícia, Kelen, Marcelo, Eleandro, Victor, Jadir, Keite, Jesus, Rosa, Eliane, Tiago, Karen, Junior, Fernanda, Izabel, Guedes, vovô Nemésio e vovó Oliva. 


\section{Resumo}

Pedro-Silva, Ronaldo; Epprecht, Eugenio Kahn (Orientador). Simulação e Análise do Desempenho de um Laboratório Analítico em um Cenário Auto-sustentável. Rio de Janeiro, 2007. 129p. Dissertação de Mestrado Departamento de Engenharia Industrial. Pontifícia Universidade Católica do Rio de Janeiro.

Os laboratórios analíticos de universidades e instituições de pesquisa privadas sem fins lucrativos estão expostos a um ambiente competitivo bem como à descontinuidade e contingenciamento de recursos financeiros oferecidos pelos órgãos de fomento. Objetivando a auto-sustentabilidade operacional, esses laboratórios estão buscando fontes de recursos próprios com a oferta crescente de prestação de serviços especializados a diferentes setores industriais. Contudo, as atividades de prestação de serviço são distintas quando comparadas às atividades de P\&D\&I, pois as primeiras são caracterizadas pela necessidade imediata ou de curto prazo e as últimas são caracterizadas pelo longo prazo. Neste contexto, torna-se necessário avaliar o trade-off entre ambientes competitivos $\mathrm{e}$ cooperativos (serviços e pesquisa) e estabelecer estratégias competitivas sustentáveis, além de práticas e técnicas de gestão que garantam a eficiência operacional sem prejuízo da qualidade e do nível de atendimento do laboratório analisado. O objetivo geral deste trabalho é, portando, analisar o desempenho do LabDRX da PUC-Rio em um cenário auto-sustentável através de um modelo de simulação de eventos discretos. Conclui-se que em tal cenário o desempenho do Laboratório atende a política de atendimento acordada com as categorias de clientes, desde que se faça a implementação conjunta de limitação dos pedidos de urgência na disciplina de fila do sistema e do aumento de capacidade com uma proposta de inovação em processo, o Distance Rietveld. A metodologia apresentada neste trabalho pode ser adaptada para outros laboratórios semelhantes que procurem sustentabilidade operacional através de prestação de serviços tecnológico para indústria.

\section{Palavras-chaves}

Simulação; laboratório analítico; difração de raios-X; gestão de laboratórios de P\&D; auto-sustentável. 


\section{Abstract}

Pedro-Silva, Ronaldo; Epprecht, Eugenio Kahn (Advisor). Simulation and Analysis of the Performance of an Analytical Laboratory in a Self-sustaining Scenario. Rio de Janeiro, 2007. 129p. MSc. Dissertation Departamento de Engenharia Industrial. Pontifícia Universidade Católica do Rio de Janeiro.

Private non-profit universities and research institutes have their analytical laboratories exposed to a competitive environment, as well as to discontinuity and limitation of financial resources offered by funding agencies. Aiming to operational self-sustainability, these laboratories are searching complementary resources by increasing the offer of specialized services to different industrial sectors. However, service activities are distinct from R\&D\&I activities, because the former are characterized by immediate or short-term need and the latter, by long-term need. In this context, it becomes necessary to evaluate the trade-off between competitive and cooperative environments (services and research), and to establish sustainable competitive strategies, as well as management practices and techniques that assure operational efficiency without quality losses or decrease in the service level. The objective of this work is, therefore, to analyze the performance of LabDRX at PUC-Rio in a self-sustainable scenario through discrete-event simulation. It was found that, in the self-sustainable scenario considered, the performance of the Laboratory satisfies the contracting policy agreed with the customer categories, as long as one makes simultaneous implementation of a limited urgent queue discipline and a capacity increase through an innovation proposal: Distance Rietveld. The methodology presented in this work can be adapted for many other similar laboratories searching for operational sustainability by offering technological services to industry.

\section{Keywords}

Simulation; analytical laboratory; X-ray diffraction; management of R\&D laboratories; self-sustaining. 


\section{Sumário}

1. Introdução 15

1.1. Contextualização 15

1.2. Objetivos geral e específicos $\quad 18$

1.3. Delimitações do estudo 18

1.4. Relevância da pesquisa 19

$\begin{array}{ll}\text { 1.5. Estrutura da dissertação } & 20\end{array}$

$\begin{array}{ll}\text { 1.6. Terminologia } & 21\end{array}$

2. Reflexões sobre a Estrutura do Setor das Universidades e Instituições Privadas de P\&D\&I $\quad 23$

2.1. Novos entrantes 25

2.2. Rivalidade entre concorrentes existentes 26

2.3. Pressões de produtos e serviços substitutos 28

2.4. Poder de negociação dos fornecedores 28

2.5. Poder de negociação dos compradores 29

2.6. Posicionamento estratégico da UIPs 29

2.7. Comentários finais $\quad 32$

3. Demanda por Serviços Tecnológicos 33

3.1. Fatores econômico-financeiros $\quad 33$

3.2. Fatores sócio-ambientais $\quad 34$

3.3. Fatores políticos-legais 35

3.4. Fatores tecnológicos 35

3.5. Prestação de serviços tecnológicos vs atividades de P\&D\&I 37

3.6. Dificuldades na contratação de serviços tecnológicos 38

4. Aplicações Industriais de Difração de Raios-X 40

4.1. Difração de raios-X $\quad 40$

4.2. Fontes de radiação e de difração de raios-X 41

4.3. A banalização das ferramentas de pesquisa 43

4.4. Método de Rietveld 45

4.5. Aplicações industriais 46

4.6. Considerações finais $\quad 51$

5. Modelo de Simulação

5.1. LabDRX - Laboratório de Difração de Raios-X 52

5.2. Demanda corrente e categorias de clientes $\quad 54$

5.3. Diagrama de Ciclo de Atividades do LabDRX 58

5.4. Custos e ponto de equilíbrio operacional do LabDRX 60

5.5. Coleta de dados $\quad 63$

5.6. Análise e tratamento dos dados 65

5.6.1. Outliers $\quad 65$

$\begin{array}{ll}\text { 5.6.2. Análise de correlação } & 67\end{array}$

5.6.3. Identificação e seleção das distribuições para o modelo 68 
5.7. Construção e descrição do modelo de simulação 70

5.8. Verificação

5.9. Validação 78

5.10. Descrição dos cenários $\quad 88$

$\begin{array}{lr}\text { 5.10.1. Cenário atual } & 89\end{array}$

$\begin{array}{lr}\text { 5.10.2. Cenário pessimista } & 90\end{array}$

$\begin{array}{lr}\text { 5.10.3. Cenário otimista } & 90\end{array}$

5.10.4. Cenário "aumento de capacidade" 91

5.10.5. Um cenário inovador, o Distance Rietveld 92

5.10.6. Cenário "urgência limitada" 95

5.10.7. Considerações no cálculo da taxa de utilização 96

6. Análise dos Resultados 100

7. Conclusões e Sugestões 106

7.1. Conclusões 106

$\begin{array}{ll}\text { 7.2. Sugestões } & 109\end{array}$

$\begin{array}{ll}\text { Bibliografia } & 111\end{array}$

Anexo A - Formulário para Coleta de Dados 116

$\begin{array}{ll}\text { Anexo B - Distribuições } & 117\end{array}$

$\begin{array}{ll}\text { Anexo C - Verificação do Modelo } & 118\end{array}$

Apêndice I - Algoritmo Simplificado do Modelo Sistema LabDRX 119 


\section{Lista de tabelas}

Tabela 1: Orçamento dos Fundos Setoriais (MCT): Reserva de Continência, 2003 a 2006

Tabela 2: Dificuldades na Contratação de Serviços Tecnológicos

Tabela 3: Crescimento nos Investimentos Mapeados (2007-2010/20022005)

Tabela 4: Pedido de urgência por categoria de clientes do LabDRX

Tabela 5: Política de prioridades do LabDRX

Tabela 6: Avaliação do impacto de retirada do outlier

Tabela 7: Comparação das médias e desvio padrão dos dados de 2005 e 2006

Tabela 8: Distribuições das variáveis de entrada

Tabela 9: Determinação da precisão dos parâmetros de desempenhos globais

Tabela 10: Validação da simulação do cenário atual

Tabela 11: Validação do cenário atual com teste de hipótese

Tabela 12: Política de atendimento por categoria de cliente em dias corridos

Tabela 13: Cenário atual

Tabela 14: Cenário pessimista

Tabela 15: Cenário otimista

Tabela 16: Cenário capacidade aumentada

Tabela 18: Cenário urgência limitada 


\section{Lista de figuras}

Figura 1: Natureza Jurídica das Instituições Ofertantes de Serviços Tecnológicos

Figura 2: Evolução das Exportações Brasileiras: 1996 a 2006

Figura 3: Difração de raios-X (a) interferência construtiva (b) interferência destrutiva

Figura 5: Mercado Brasileiro de Catalisadores FCC - de Leito Fluidizado e HDR - Hidrorefino

Figura 6: Aplicação da difração de raios-X na caracterização de nantubos de titanato: (a) difratogramas de TiTanate Nano Tubos/sódio Hexatitanato (TTNT/H), (b) Imagem de uma amostra de nanotubo de titanato $(\mathrm{TTNT} / \mathrm{H})$ a $200^{\circ} \mathrm{C}$

Figura 7: Número de análises realizadas e nos últimos 5,5 anos no LabDRX

Figura 8: Número de análise solicitadas ao LabDRX nos últimos 2 anos

$\begin{array}{ll}\text { Figura 9: Diagrama de Ciclo de Atividade do Sistema LabDRX } & 58 \\ \text { Figura 10: Demanda corrente do sistema LabDRX } & 64\end{array}$

Figura 11: Gráfico box-plot para identificação de outliers. 65

Figura 12: Gráfico de Dispersão para Verificação da Presença de 67 Autocorrelação de 1 Período ("lag 1") de Cada uma das 4 Variáveis de Interesse Figura 13: Modelo do Sistema LabDRX

Figura 14: Submodelo Controle do Relógio

Figura 15: Submodelo Chegada de Requisições 72

Figura 16: Submodelo Características dos Clientes $\quad 73$

Figura 17a: Submodelo Difratômetro de Raios-X 74

Figura 17b: Submodelo Difratômetro de Raios-X (continuação) 75

Figura 17c: Submodelo Difratômetro de Raios-X (continuação) 75

Figura 18: Submodelo Análise Qualitativa e Quantitativa 76

Figura 19: Submodelo Lead Time 77

Figura 20: Evolução da Taxa de Utilização do DRX (Goniômetro 1) em uma Simulação Piloto com 5 Replicações de 8760 horas cada, para Identificação do Período de Steady-State e Warm-up de 500 horas (aprox.: 20 dias)

Figura 21: Planejamento da simulação de cenários

Figura 22: Diagrama de Ciclo de Atividade do Sistema LabDRX com Distance Rietveld e Rietveld on-line

Figura 23: Comparação da taxa de utilização do difratômetro de raios-X $(\rho)$ com a demanda prospectiva para cada cenário.

Figura 24: Relacionamento entre taxa de utilização do serviço e a qualidade do serviço

Figura 25: Desempenho do sistema LabDRX diante da categoria de clientes outras instituições de P\&D\&I.

Figura 26: Desempenho do sistema LabDRX diante da categoria de clientes outros departamentos da PUC-Rio 
Figura 27: Desempenho do sistema LabDRX diante da categoria de clientes DCMM
Figura 28: Desempenho do sistema LabDRX diante da categoria de clientes empresas

Figura 29: Desempenho do sistema LabDRX diante dos pedidos de urgência de todas as categoria de clientes

Figura 30: Efeito da urgência limitada na categoria de clientes 
Science would soon come to a standstill if all scientists were of the same intellectual type. Science needs scholars with many different talents.

Max von Laue, Fifty years of X-ray Diffraction 